ISSN 1112-9867

http://www.jfas.info

\title{
UNSTEADY MHD FLOW ALONG EXPONENTIALLY ACCELERATED VERTICAL FLAT SURFACE THROUGH POROUS MEDIUM WITH VARIABLE TEMPERATURE AND HALL CURRENT IN A ROATATING SYSTEM
}

\author{
U.S. Rajput ${ }^{1}$ and M. Shareef ${ }^{2 *}$
}

Department of Mathematics and Astronomy, University of Lucknow, Lucknow-226007, India

Received: 27 December 2016 / Accepted: 28 April 2017 / Published online: 01 May 2017

\begin{abstract}
The present study is carried out to examine the unsteady flow along a non-conducting vertical exponentially accelerated infinitely long plate with heat source and Hall current through a porous medium in a rotating system. The non-dimensional governing equations of model are solved by using Integral transform technique. The results are analysed with the help of graphs and tables. It is noticed that the flow pattern is affected significantly with plate acceleration, Hall current, radiation, porous medium and heat source. The outcomes of the study may find applications in various fields related to the solar physics dealing with the solar cycle, the sunspot development, the structure of rotating magnetic stars etc.
\end{abstract}

Keywords: MHD; Rotation; Porous medium; Hall Current.

Author Correspondence, e-mail: rajputshareeflu@mail.com

doi: http://dx.doi.org/10.4314/jfas.v9i2.28

\section{INTRODUCTION}

The fluid flow along infinite flat surface is a classical problem of the fluid dynamics. The application of MHD viscous, incompressible unsteady flow involving radiation and heat source have been found in many areas of engineering and science. Steworten $[1,2]$ had done 
significant study to understand the behaviour of the fluids in unsteady boundary layer. His study was completely based on boundary layer theory. The strength of magnetic field can stabilizes such flow within porous and non-porous medium. Therefor the results of the study has significant role in the designing of heat exchangers, MHD pumps, MHD generator, nuclear reactors, oil exploration etc. Thus various researches have been made in this field like, mass transfer and radiation effect on 2-D flow past an impulsively started infinitely long vertical surface was studied by Prasad et al. [3]. They used finite-difference method to solve the partial differential equations of the model and analysed that the velocity of the fluid decreases in the vinicity of plate when the radiation is increased. Sharidan et al. [4] analysed the unsteady Magnetohydrodynamic convective flow past an infinite inclined plate through a porous medium with radiation. They obtained analytical solution by using Laplace transform and found that the radiation parameter retards the fluid flow in the region near the plate. Also, the effect of chemical reaction and heat absorption/generation on such a flow was analysed by Chamka [5]. He solved the problem analytically and pointed that magnetic field, Prandlt number \& Schmidt number retard the fluid velocity. Makinde and Mhone [6] examined such flow by taking conducting optically thin fluid with variable wall temperature.

Further, if we use the strong magnetic field, the Hall effect can not be neglected. Also, the rotating flow of electrically conducting fluid has attracted many scholars due to their abundant applications in the field like geophysical and astrophysical etc. For instance, Agarwal et al. [7] analyzed the effect of dissipation and Hall current on free convective MHD flow. It was observed by Agarwal et al. [7] that shear-stresses at the surface along primary \& secondary directions increases and decreases, respectively, with the increase in Hall parameter and magnetic field. Mazumdar et al. [8] worked on flow with heat transfer in the hydrodynamic Ekman layer on a porous plate with Hall effects. Further, Jaimala et al. [9] analysed the effect of Hall current on an electrically conducting couple-stress fluid layer heated from below. In 2014, Reddy [10] worked on MHD flow along vertical plate with radiation and chemical reaction. He [10] found that the chemical reaction retards the fluid velocity, decreases the concentration and increases the thermal boundary layer thickness. Recently Mahmoudpour Molaei et al. [11] consider the model having non-newtonian power-law fluid over a vertical 
surface with suction effects.

The model under consideration analyzes an unsteady flow along an accelerated non-conducting vertical flat surface of infinite extent with heat source and radiation through a porous medium in a rotating system. The solution of the PDEs involved in the model is found with the help of Laplace transform. The effect of various parameters on the flow field are analysed and discussed by ploting graphs against boundary layer co-ordinat. Also the variation in Nusselt number \& skin-friction are shown by numerical values in tables.

\section{MATHEMATICAL FORMULATION}

Consider a flow of incompressible, viscous and electrically conducting fluid past an infinite vertical non-conducting flat surface in porous medium. Let the plate is moving vertically upward along $x^{\prime}-$ axis and $z^{\prime}-$ axis is chosen perpendicular to $x^{\prime}$-axis. Also the system is rotating with a uniform angular velocity $\Omega^{\prime}$ about $z^{\prime}-$ axis with an applied uniform magnetic field $B_{o}$ perpendicular to the surface as shown in Figure - (a). Here we also assume that the fluid has low magnetic Reynolds number, so we neglect the induced magnetic field, which is comparatively very small generated by motion of conducting fluid, therefore $\overrightarrow{\mathbf{B}}=\left(0,0, B_{o}\right)$. Also it is assumed that no applied \& polarization voltage exist, so induced electric field $\overrightarrow{\mathbf{E}}=(0,0,0)$. Due to infinite extent of the plate all the physical variables are considered to be the function of $\mathrm{z}^{\prime}$ and $t^{\prime}$ only. 


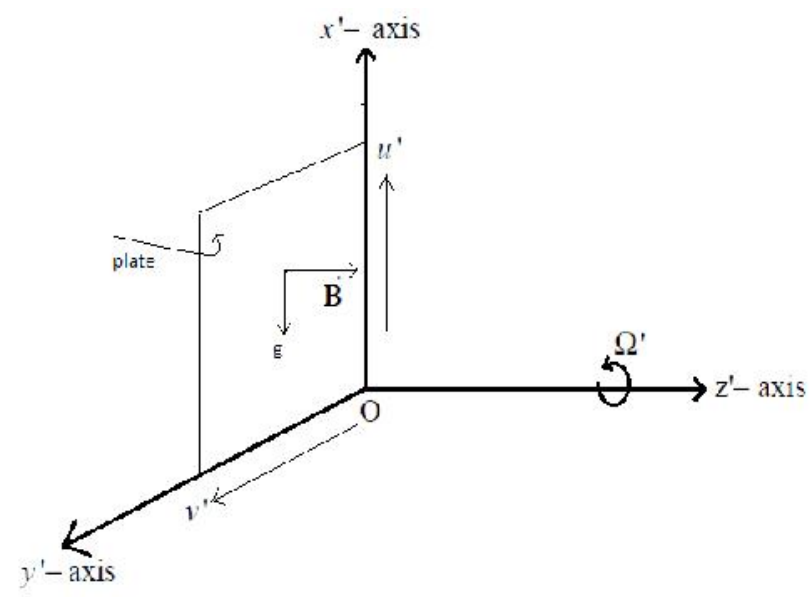

Fig. (a)

Initially, the system is at rest having a uniform temperature $T_{o}$. At time $t^{\prime}>0$, the plate started to move with a velocity $u_{o} e^{-c^{\prime} t^{\prime}}$ in the positive $x^{\prime}$-direction and the plate temperature is raised to $T_{p}$. The motion of the plate and the free convection causes the disturbance in the fluid. From the equation of conservation of electric charge $\nabla . \overrightarrow{\mathbf{J}}$, we have $J_{z^{\prime}}=$ constant, where $\overrightarrow{\mathbf{J}}=\left(J_{x^{\prime}}, J_{y^{\prime}}, J_{z^{\prime}}\right)$ is the current density vector. As the plate under consideration is non-conducting, therefore, at the plate $J_{z^{\prime}}=0$. Thus $J_{z^{\prime}}=0$ everywhere in the fluid. So, the PDEs of the model with Boussinesq's approximations are as follows:

$$
\begin{aligned}
& \frac{\partial u^{\prime}}{\partial t^{\prime}}-2 \Omega^{\prime} v^{\prime}=v \frac{\partial^{2} u^{\prime}}{\partial z^{\prime 2}}+g \beta\left(T-T_{0}\right)+\frac{B_{o}}{\rho} J_{y^{\prime}}-\frac{v}{K^{\prime}} u^{\prime}, \\
& \frac{\partial v^{\prime}}{\partial t^{\prime}}+2 \Omega^{\prime} u^{\prime}=v \frac{\partial^{2} v^{\prime}}{\partial z^{\prime 2}}-\frac{B_{o}}{\rho} J_{x^{\prime}}-\frac{v}{K^{\prime}} v^{\prime}, \\
& \frac{\partial T}{\partial t^{\prime}}=\frac{k}{\rho c_{p}} \frac{\partial^{2} T}{\partial z^{\prime 2}}-\frac{1}{\rho c_{p}} \frac{\partial q^{(\mathrm{r})}}{\partial z^{\prime}}+\frac{Q_{o}}{\rho c_{p}}\left(T_{p}-T_{o}\right),
\end{aligned}
$$

The boundary conditions taken are as under:

$$
\left.\begin{array}{l}
t^{\prime} \leq 0: u^{\prime}=0, v^{\prime}=0, T=T_{o} \quad \forall z^{\prime}, \\
t^{\prime}>0: u^{\prime}=u_{o} \mathrm{e}^{-c^{\prime} t^{\prime}}\left(c^{\prime}>0\right), v^{\prime}=0, T=T_{o}+\left(T_{p}-T_{o}\right) \frac{u_{o}^{2}}{v} t^{\prime}, \text { at } z^{\prime}=0, \\
u^{\prime} \rightarrow 0, v^{\prime} \rightarrow 0, T \rightarrow T_{o}, \text { as } \quad z^{\prime} \rightarrow \infty .
\end{array}\right\}
$$


Neglecting the electron pressure gradient, the ion slip and the thermo-electric effects, the generalised Ohm's law with Hall effect is given as

$$
\overrightarrow{\mathbf{J}}+\frac{\omega_{e} \tau_{e}}{B_{o}}(\overrightarrow{\mathbf{J}} \times \overrightarrow{\mathbf{B}})=\sigma(\overrightarrow{\mathbf{E}}+\overrightarrow{\mathbf{q}} \times \overrightarrow{\mathbf{B}})
$$

On solving (5), we get $J_{x^{\prime}}=\frac{\sigma B_{o}\left(v^{\prime}+m u^{\prime}\right)}{1+m^{2}}, J_{y^{\prime}}=\frac{\sigma B_{o}\left(m v^{\prime}-u^{\prime}\right)}{1+m^{2}}$

By using Rosseland approximation (Brewster [12]), the heat flux due to radiation $q^{(\mathrm{r})}$ is given by

$$
q^{(\mathrm{r})}=-\frac{4 \sigma_{s}}{3 k_{e}} \frac{\partial T^{\prime 4}}{\partial z^{\prime}}
$$

Here $u^{\prime}$ and $v^{\prime}$ are the primery and secondary velocity respectively, $t^{\prime}-$ time, $T$-temperature at any time $t^{\prime}, u_{o}$-velocity parameter, $c^{\prime}$ - exponential parameter, $K^{\prime}-$ permeability parameter, $\quad \beta$-volumetric thermal expansion coefficient, $q^{(\mathrm{r})}$-radiative heat flux, $\alpha$-thermal diffusivity, $k_{e}$-mean absorption coefficient, $\omega_{e}$-cyclotron frequency of electron, $\tau_{e}$-electron collision time, $g$-gravitational acceleration, $\rho$-density of fluid, $v-$ kinematic viscosity, $\sigma_{s}-$ Stefan-Boltzmann constant, $m\left(=\omega_{e} \tau_{e}\right)-$ Hall parameter, $J_{x^{\prime}}-$ current density along $x^{\prime}-$ axis and $J_{y^{\prime}}-$ current density along $y^{\prime}-$ axis.

If the temperature variation within the flow is sufficiently small, then by Taylor series exapansion of $T^{14}$ about $T_{o}$ (neglecting higher order terms), we get,

$$
T^{4} \cong 4 T_{o}^{3} T-3 T_{o}^{4}
$$

By using above results given in equations (6) and (7), equation (3) becomes

$$
\frac{\partial T}{\partial t^{\prime}}=\alpha \frac{\partial^{2} T}{\partial z^{\prime 2}}+\frac{16 \sigma \alpha T_{o}^{3}}{3 k_{e} k} \frac{\partial^{2} T}{\partial z^{\prime 2}}+\frac{Q_{o}}{\rho c_{p}}\left(T-T_{o}\right)
$$

Or $\quad \frac{\partial T}{\partial t^{\prime}}=\alpha\left(1+\frac{4}{3 N}\right) \frac{\partial^{2} T}{\partial z^{\prime 2}}+\frac{Q_{o}}{\rho c_{p}}\left(\bar{T}-\bar{T}_{\infty}\right)$

Dimensionless partial differential equations can be obtaine by using the following non-dimensional parameters: 


$$
\begin{aligned}
& u=\frac{u^{\prime}}{u_{o}}, v=\frac{v^{\prime}}{u_{o}}, t=\frac{u_{o}^{2}}{v} t^{\prime}, z=\frac{u_{o}}{v} z^{\prime}, \theta=\frac{\left(T-T_{o}\right)}{\left(T_{p}-T_{o}\right)}, \mathrm{P}_{r}=\frac{v}{\alpha}, R=\frac{k_{e} k}{4 \sigma_{s} T_{o}^{3}}, \\
& M^{2}=\frac{\sigma B_{o}^{2} v}{\rho u_{o}^{2}}, \mathrm{c}=\frac{v}{u_{o}^{2}} \mathrm{c}^{\prime}, \Omega=\frac{v}{u_{o}^{2}} \Omega^{\prime}, K=\frac{u_{o}^{2}}{v^{2}} K^{\prime}, G_{r}=\frac{g \beta v\left(T_{p}-T_{\infty}\right)}{u_{o}^{3}} .
\end{aligned}
$$

Here $u$ and $v$ are the dimensionless primary \& secondary velocities respectvely, $z$-dimensionless spatial coordinate, $\theta$-dimensionless temperature, $P_{r}$-Prandlt number, $G_{r}$ - Thermal Grashof number, $t$ - dimensionless time, $\Omega$ - dimensionless rotation parameter, $R$-Radiation parameter and $M$ - magnetic field parameter.

Using equation (9), equations (1), (2), (8) and (4) respectively, become:

$$
\begin{aligned}
& \frac{\partial u}{\partial t}-2 \Omega v=\frac{\partial^{2} u}{\partial z^{2}}+\frac{M^{2}}{\left(1+m^{2}\right)}(m v-u)+G_{r} \theta-\frac{u}{K}, \\
& \frac{\partial v}{\partial t}+2 \Omega u=\frac{\partial^{2} v}{\partial z^{2}}-\frac{M^{2}}{\left(1+m^{2}\right)}(v+m u)-\frac{v}{K}, \\
& \frac{\partial \theta}{\partial t}=\frac{1}{R_{a} P_{r}} \frac{\partial^{2} \theta}{\partial z^{2}}+Q \theta . \\
& t \leq 0: u=0, v=0, \theta=0, \quad \forall z, \\
& \left.t>0: u=e^{-c t}, v=0, \theta=t, \quad \text { at } z=0,\right\} \\
& u \rightarrow 0, v \rightarrow 0, \theta \rightarrow 0, \text { as } z \rightarrow \infty \text {. }
\end{aligned}
$$

To solve above system, assume $\mathbf{V}=u+i v$. Then using equations (10) and (11), we get,

$$
\frac{\partial \mathbf{V}}{\partial t}=\frac{\partial^{2} \mathbf{V}}{\partial z^{2}}-b \mathbf{V}+G_{r} \theta
$$

And equation (13) is transformed:

$$
\left.\begin{array}{l}
t \leq 0: \mathbf{V}=0, \theta=0, \quad \forall z, \\
t>0: \mathbf{V}=e^{-c t}, \theta=t, \text { at } z=0, \\
\mathbf{V} \rightarrow 0, \theta \rightarrow 0, \quad \text { as } z \rightarrow \infty .
\end{array}\right\}
$$

The equations (12) \& (14) with the above boundary conditions prescribed in equation (15) are solved with the help of Laplace transform method.

And the solution is as under:

$$
\mathbf{V}(z, t)=a_{1} e^{-B_{1} t}\left\{-2 \cosh \left(a_{3} z\right)+e^{-a_{3} z} \operatorname{Erf}\left(\eta-a_{3} \sqrt{t}\right)+e^{a_{3} z} \operatorname{Erf}\left(\eta+a_{3} \sqrt{t}\right)\right\}-2\left(a_{1}-a_{12} t\right) \cosh \left(a_{7} z\right)
$$




$$
\begin{aligned}
& +\left(a_{1}-a_{1} B_{1} t+a_{4} z\right) e^{-a_{2} z} \operatorname{Erf}\left(a_{2} \sqrt{t}-\eta\right)+\left(-a_{1}+a_{1} B_{1} t+a_{4} z\right) e^{a_{2} z} \operatorname{Erf}\left(a_{2} \sqrt{t}+\eta\right) \\
& -\left(a_{1}-a_{12} t+a_{6} z\right) e^{-a_{7} z} \operatorname{Erf}\left(a_{8} \sqrt{t}-a_{9} \eta\right)+\left(a_{1}-a_{12} t-a_{6} z\right) e^{a_{7} z} \operatorname{Erf}\left(a_{8} \sqrt{t}+a_{9} \eta\right) \\
& +a_{1} e^{-B_{1} t}\left\{2 \cosh \left(a_{10} z\right)+e^{-a_{10} z} \operatorname{Erf}\left(a_{11} \sqrt{t}-a_{9} \eta\right)-e^{a_{10} z} \operatorname{Erf}\left(a_{11} \sqrt{t}+a_{9} \eta\right)\right\} \\
& +2 a_{1}\left(1-B_{1} t\right)\left\{\cosh \left(a_{2} z\right)\right\}-\frac{1}{2} e^{a_{5} z-c t} \operatorname{Erf}\left(a_{5} \sqrt{t}+\eta\right)+2 a_{6} z \sinh \left(a_{7} z\right) \\
\theta(z, t)= & a_{13} z \sinh \left(a_{7} z\right)-\left(a_{14} z+a_{16} t\right) \mathrm{e}^{a_{7} z} \operatorname{Erf}\left(a_{8} \sqrt{t}+a_{9} \eta\right) \\
& +a_{15} t \cosh \left(a_{7} z\right)+\left(a_{16} t-a_{14} z\right) \mathrm{e}^{-a_{7} z} \operatorname{Erf}\left(a_{8} \sqrt{t}-a_{9} \eta\right)
\end{aligned}
$$

The dimensionless skin-friction components $\tau_{x}, \tau_{y}$ and Nusselt number $N u$ are obtained as:

$$
\begin{aligned}
\tau_{x}+i \tau_{y}=-\left.\frac{\partial \mathbf{V}}{\partial z}\right|_{z=0} & =\left(b_{1}-b_{3}-b_{5} t\right) \operatorname{Erf}\left(a_{2} \sqrt{t}\right)+\left(b_{6}-b_{8}-b_{10} t\right) \operatorname{Erf}\left(a_{8} \sqrt{t}\right)+b_{9} \sqrt{t} e^{Q t} \\
& +e^{-B_{1} t}\left(b _ { 7 } \operatorname { E r f } \left(a_{11} \sqrt{t}-b_{2} \operatorname{Erf}\left(a_{3} \sqrt{t}\right)+e^{-b t}\left(\frac{1}{\sqrt{\pi t}}-b_{4} \sqrt{t}\right)+a_{5} e^{-c t} \operatorname{Erf}\left(a_{5} \sqrt{t}\right)\right.\right.
\end{aligned}
$$$$
N u=-\left.\frac{\partial \theta}{\partial z}\right|_{z=0}=-\left(b_{11}+b_{7} t\right) \operatorname{Erf}\left(a_{8} \sqrt{t}\right)-b_{12} \sqrt{t} e^{Q t}
$$

\section{RESULTS AND DISCUSSION}

In order to explain the significance of the study the variation in the temperature and velocity within the boundary layer for different parameters involved is shown in Figures 1 - 10. It is noticed from figures 1 to 7 that magnitude of secondary velocity $v$ attains a maximum value near the plate and then gradually decreases while primary velocity $u$ decreases continuously on increasing coordinate $z$. Figures 1 and 2 shows the variation in the velocity of the fluid with acceleration parameter $\mathrm{c}$ at different time $(\mathrm{t}=0.3$ and $\mathrm{t}=0.6)$. And it is noticed that both the components of velocity decrease with c but as the time increases the velocities decreases comparatively slow rate. Effect of Q on fluid velocity is shown in Figures 3 and 4 . It is examine that at a given time, $\mathrm{Q}$ increases the velocities in boh the directions and as the time increases the velocities increases rapidly with $Q$. The velocity variation with Hall current is display in Figure 5, which shows that on increasing Hall current parameter, the primary velocity increases, while the secondary velocity decreases rapidly. This shows that the Hall current parameter $m$ accelerate and deaccelerate the primery and secondary velocities 
respectively. The behavior of velocity with radiation parameter $R$ is shown by Figure 6, which display that it slow down the velocities in both the directions. Figure 7 show the consequence of the rotation on the folw field and it is analysed that the rotation parameter retards the primary velocity and accelerate the secondary velocity. Figures 8 to10 show the temperature variation in the region around the plat with coordinate $z$. It can be analysed from Figure 8 and 9 that, at a perticular instant of time, the temperature in the system increases with heat source parameter Q and the increasing rate becomes high as time increases. Also, it is observed that the temperature in the system is inversely proportional to Prandlt number $P_{r}$ and radiation parameter R (Figure 10). Thus, the system cools down as $R$ and $P_{r}$ increases.

Tables 1 to 4 display the variation of skin-friction and Nusselt number for different paprameters. From table -1 , it is found that, $\tau_{x}$ increases when $M$ is increased (keeping other parameters fixed) but if values of $\mathrm{m}$ and $\mathrm{K}$ is increased, it gets decreased. Also, it is noticed that $\tau_{y}$ increases with $\mathrm{m}$ and it decreases when $\mathrm{M}$ and $\mathrm{K}$ are increased. From the table 2 and 3 it can analysed that $\tau_{x}$ increases when the values of $\Omega$ and $\mathrm{R}$ are increased while it gets decreased with c and $\mathrm{Q}$. And $\tau_{y}$ increases with $\mathrm{c}$ and $\mathrm{R}$ while it decreases when $\Omega$ and $\mathrm{Q}$ are increased. The Nusselt number decreases with Q and increases with R (Table 4).

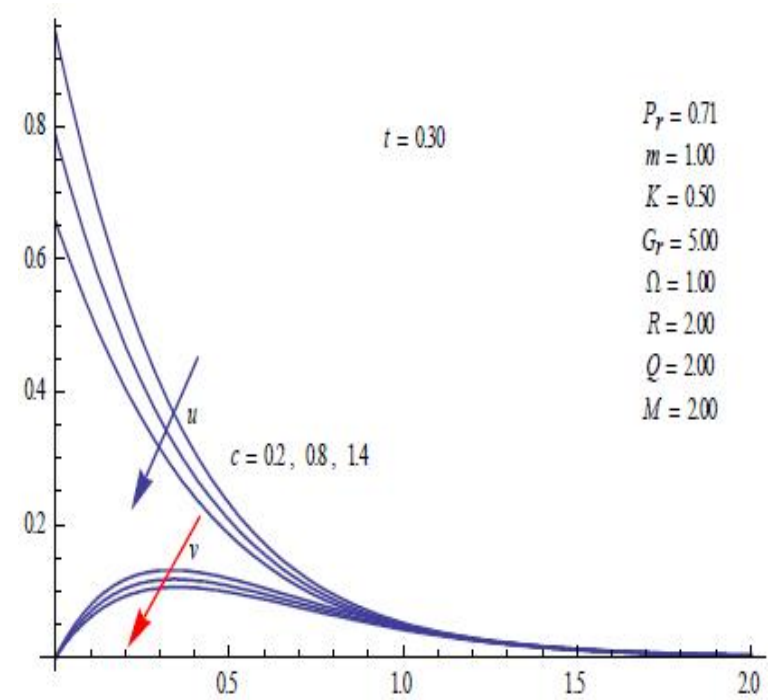

Fig.1.Velocity profile for $\mathrm{c}$ at $\mathrm{t}=0.2$

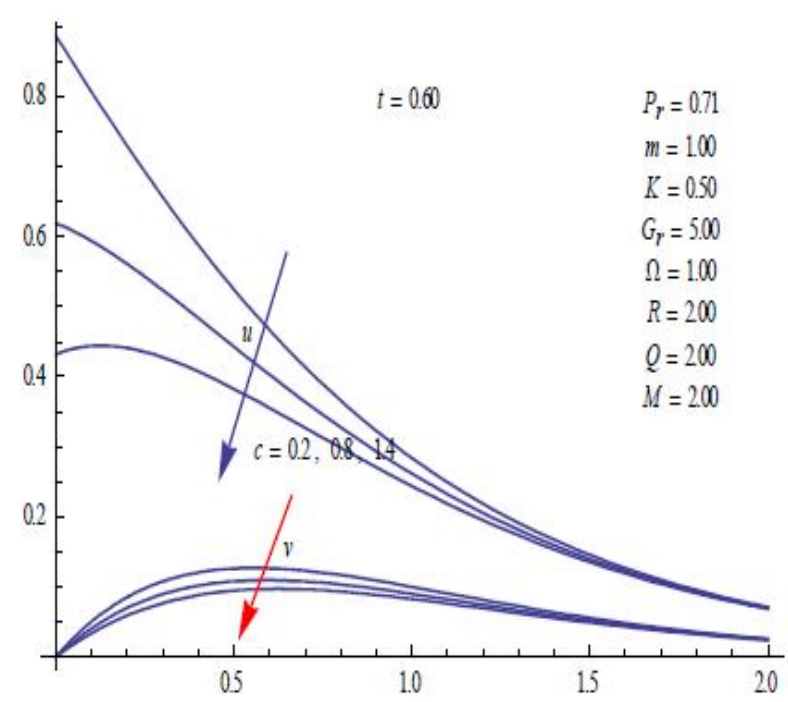

Fig.2.Velocity profile for $\mathrm{c}$ at $\mathrm{t}=0.6$ 


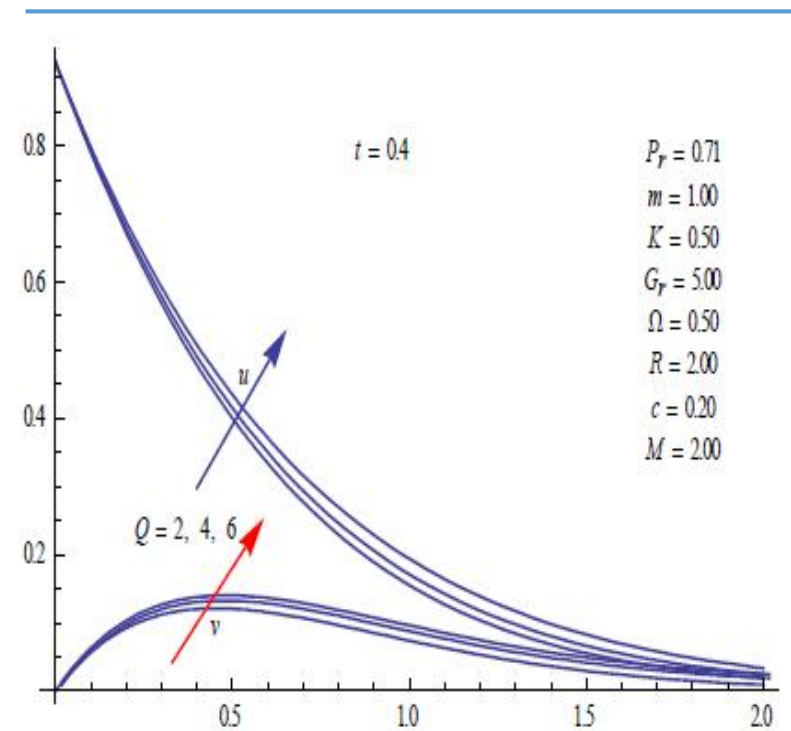

Fig.3.Velocity profile for $\mathrm{Q}$ at $\mathrm{t}=0.4$

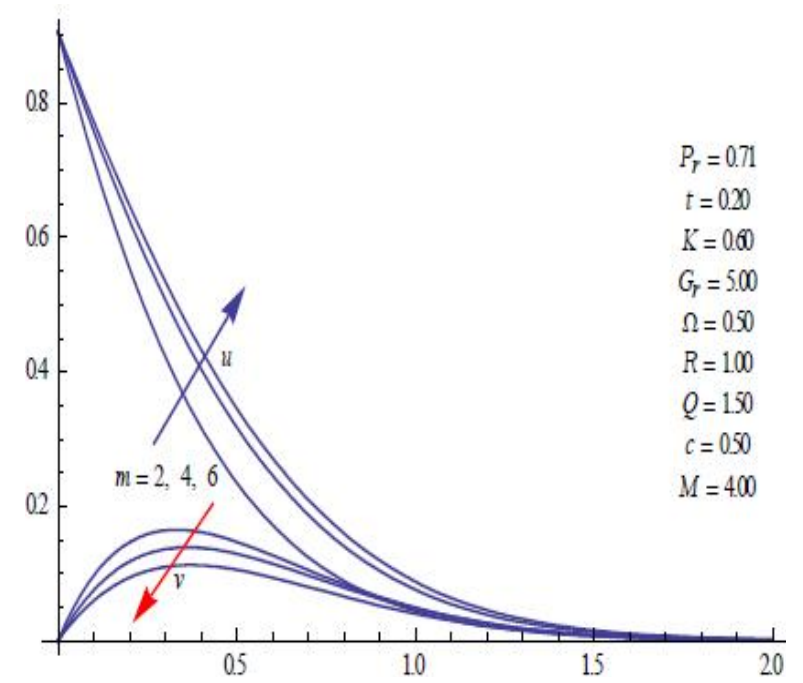

Fig.5.Velocity profile for $\mathrm{m}$

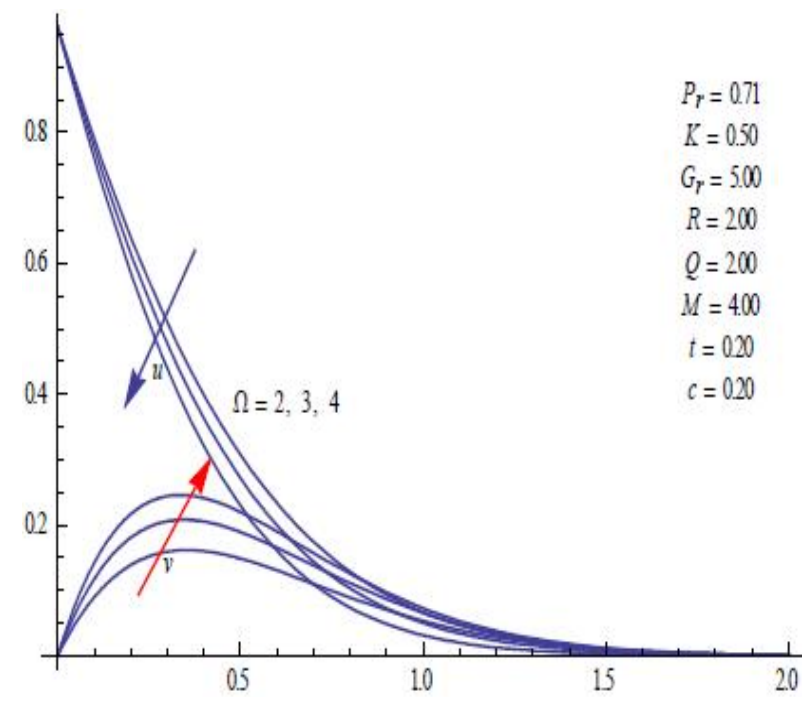

Fig.7.Velocity profile for

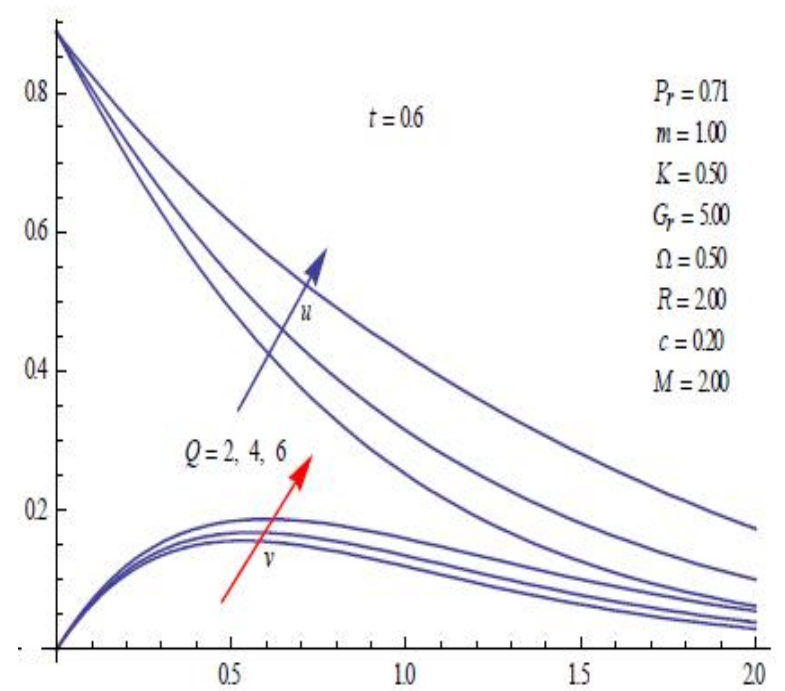

Fig.4. Velocity profile for $\mathrm{Q}$ at $\mathrm{t}=0.6$

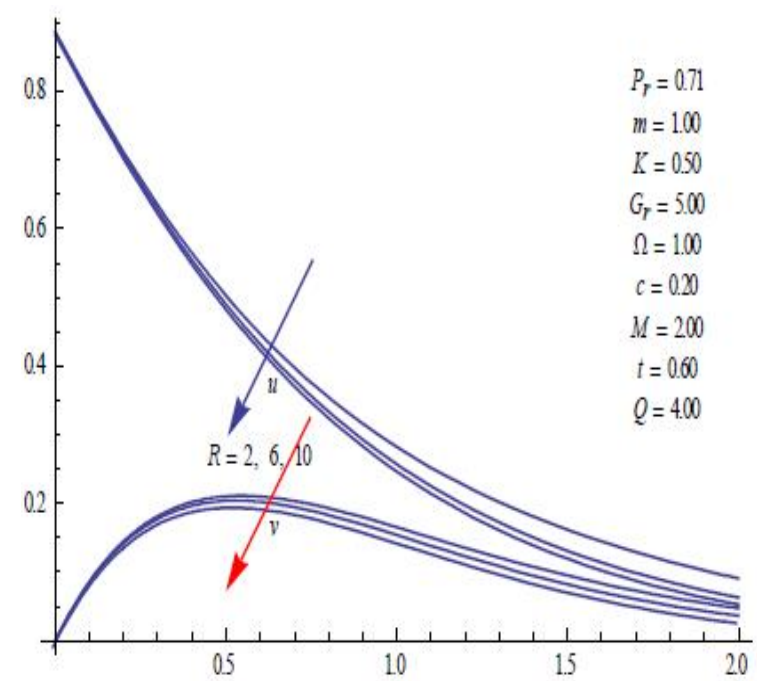

Fig.6.Velocity profile for R

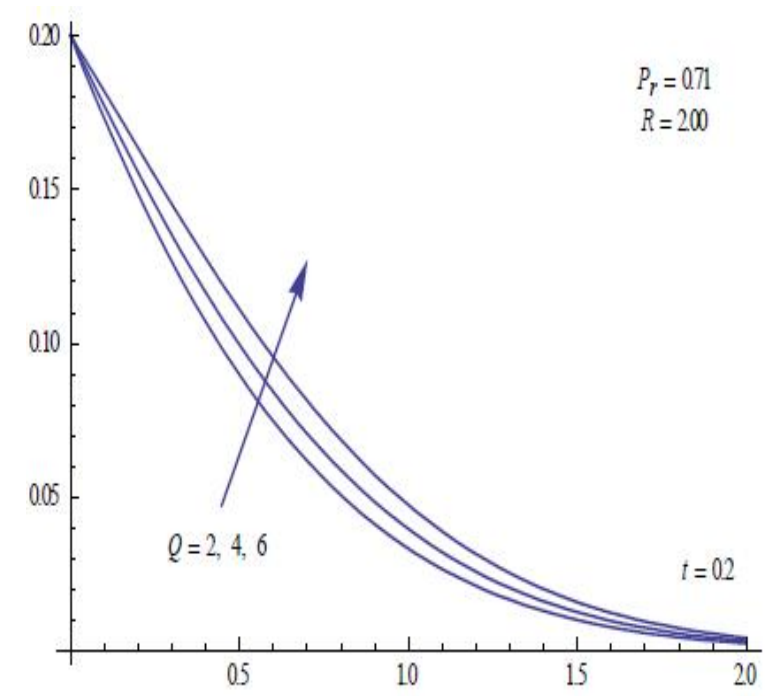

Fig.8. Temperature profile for $\mathrm{Q}$ at $\mathrm{t}=0.2$ 


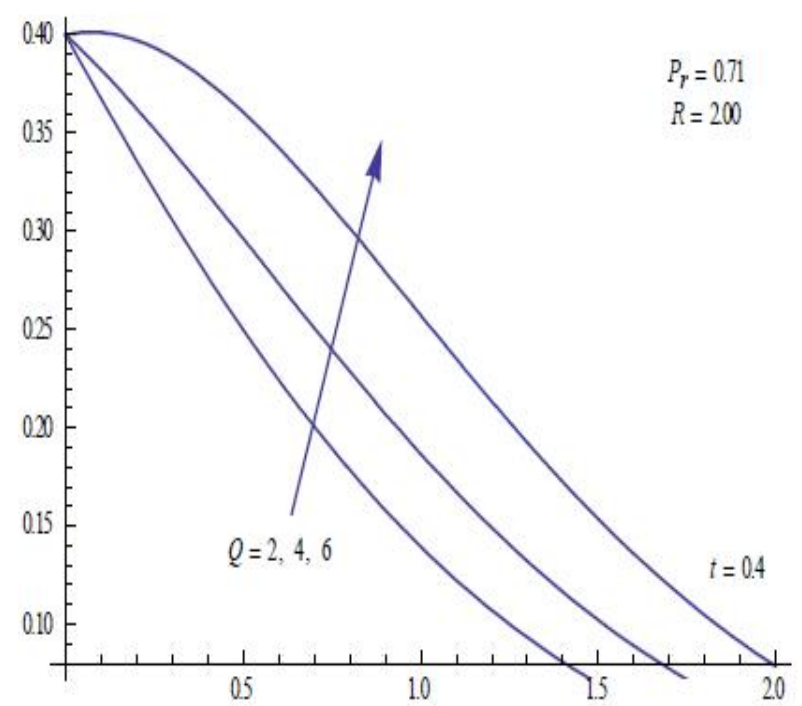

Fig.9. Teperature profile for $\mathrm{Q}$ at $\mathrm{t}=0.4$

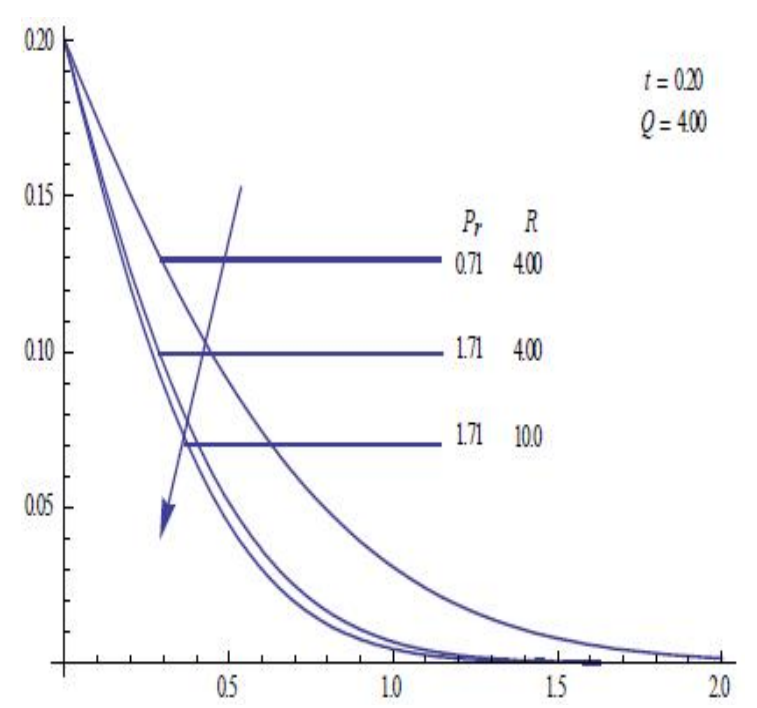

Fig.10. Temperature profile for $\operatorname{Pr} \& \mathrm{R}$

\begin{tabular}{|c|c|c|c|c|c|c|}
\hline \multicolumn{6}{|c|}{ Table.1. Skin Friction for different values of $\mathrm{m}$} \\
\hline \multirow{2}{*}{$\mathrm{t}=0.2$} & \multirow{2}{*}{$\mathrm{m}$} & \multicolumn{2}{|c|}{$\mathrm{K}=0.3$} & \multicolumn{3}{|c|}{$\mathrm{K}=0.5$} \\
\cline { 2 - 6 } & 1 & 1.951 & 0.523 & 1.713 & 0.561 \\
\hline \multirow{3}{*}{$\mathrm{M}=2$} & 2 & 1.726 & 0.484 & 1.473 & 0.521 \\
\cline { 2 - 6 } & 3 & 1.642 & 0.419 & 1.381 & 0.453 \\
\hline \multirow{3}{*}{$\mathrm{M}=3$} & 1 & 2.425 & 0.839 & 2.226 & 0.891 \\
\cline { 2 - 6 } & 2 & 1.971 & 0.801 & 1.739 & 0.859 \\
\cline { 2 - 6 } & 3 & 1.779 & 0.681 & 1.532 & 0.732 \\
\hline
\end{tabular}

\begin{tabular}{|c|c|c|c|}
\hline \multicolumn{4}{|c|}{ Table.2. Skin Friction for } \\
\hline $\mathrm{t}=0.2$ & & {$\left[\tau_{x}\right.$} & $-\tau_{y}$ \\
\hline \multirow{3}{*}{$\mathrm{c}=0.2$} & 0.2 & 1.871 & 0.469 \\
\cline { 2 - 4 } & 0.4 & 1.888 & 0.585 \\
\cline { 2 - 4 } & 0.6 & 1.901 & 0.662 \\
\hline \multirow{4}{*}{$\mathrm{c}=2.0$} & 0.2 & 0.997 & 0.365 \\
\cline { 2 - 4 } & 0.4 & 1.012 & 0.455 \\
\cline { 2 - 4 } & 0.6 & 1.023 & 0.514 \\
\hline
\end{tabular}

\begin{tabular}{|c|c|c|c|}
\hline \multicolumn{4}{|c|}{ Table.3. Skin Friction for $\mathrm{R}$} \\
\hline $\mathrm{t}=0.2$ & $\mathrm{R}$ & $\tau_{x}$ & $-\tau_{y}$ \\
\hline \multirow{3}{*}{$\mathrm{Q}=2$} & 2 & 1.713 & 0.5608 \\
\hline & 4 & 1.723 & 0.5595 \\
\hline & 6 & 1.726 & 0.5592 \\
\hline \multirow{3}{*}{$\mathrm{Q}=4$} & 2 & 1.707 & 0.5614 \\
\hline & 4 & 1.717 & 0.5601 \\
\hline & 6 & 1.719 & 0.5598 \\
\hline
\end{tabular}

\begin{tabular}{|c|c|c|}
\hline \multicolumn{3}{|c|}{ Table.4. (Nusselt Number) } \\
\hline $\mathrm{t}=0.2$ & $\mathrm{R}$ & $\mathrm{Nu}$ \\
\hline \multirow{3}{*}{$\mathrm{Q}=2$} & 2 & 0.2834 \\
\cline { 2 - 3 } & 6 & 0.3312 \\
\cline { 2 - 3 } & 10 & 0.3439 \\
\hline \multirow{3}{*}{$\mathrm{Q}=4$} & 2 & 0.2336 \\
\cline { 2 - 3 } & 6 & 0.2728 \\
\cline { 2 - 3 } & 10 & 0.2833 \\
\hline
\end{tabular}




\section{CONCLUSION}

As expected, the rotation parameter slows down the primery velocity and speed up the secondary velocity. Also, it is found that the Hall current accelerate the primary velocity and deaccelerate the secondary velocity. When the radiation parameter increases, the temperature and velocity both decreases. And at a perticular instant of time, momentum boundary layer thickness decreases with increase in c. Also, the temperature and velocity both increases with increase in Q. The skin-friction rises with the rise in radiation and rotation parameter. And it decreases with increase in $\mathrm{m}, \mathrm{c}$ and $\mathrm{Q}$. The outcomes will have applications in the research related to the solar physics dealing with the sunspot development, the solar cycle, the structure of rotating magnetic stars, and geophysics.

Appendix

$$
\begin{aligned}
& b=\frac{M^{2} i}{m+i}+2 i \Omega+\frac{1}{K}, R_{a}=\frac{3 R}{3 R+4}, A_{1}=\frac{G_{r}}{1-P_{r} R_{a}}, B_{1}=\frac{b+Q P_{r} R_{a}}{1-P_{r} R_{a}}, a_{1}=\frac{A_{1}}{2 B_{1}^{2}}, a_{2}=\sqrt{b}, \\
& a_{3}=\sqrt{b-B_{1}}, a_{4}=\frac{A_{1}}{4 B_{1} \sqrt{b}}, a_{5}=\sqrt{b-c}, a_{6}=\frac{A_{1} \sqrt{P_{r} R_{a}}}{4 B_{1} \sqrt{-Q}}, a_{7}=\sqrt{-Q P_{r} R_{a}}, a_{8}=\sqrt{-Q}, \\
& a_{9}=\sqrt{P_{r} R_{a}}, a_{10}=\sqrt{-\left(Q+B_{1}\right) P_{r} R_{a}}, a_{11}=\sqrt{-\left(Q+B_{1}\right)}, a_{12}=\frac{A_{1}}{2 B_{1}}, a_{13}=\frac{P_{r} R_{a}}{2 a_{8} a_{9}}, a_{14}=\frac{P_{r} R_{a}}{4 a_{8} a_{9}}, \\
& a_{15}=\frac{a_{7}}{a_{8} a_{9}}, a_{16}=\frac{a_{7}}{2 a_{8} a_{9}}, b_{1}=2 a_{1} a_{2}, b_{2}=2 a_{1} a_{3}, b_{3}=\frac{a_{12}}{a_{2}}, b_{4}=\frac{2 a_{12}}{\sqrt{\pi}}, b_{5}=2 a_{2} a_{12}, b_{6}=\frac{a_{9} a_{12}}{a_{8}}, \\
& b_{7}=2 a_{1} a_{9} a_{11}, b_{8}=2 a_{1} a_{9} a_{8}, b_{9}=\frac{2 a_{9} a_{12}}{\sqrt{\pi}}, b_{10}=2 a_{12} a_{9} a_{8}, b_{11}=\frac{2 P_{r} R_{a}}{2 a_{7}}, b_{12}=\frac{a_{9}}{\sqrt{\pi}}, \eta=\frac{z}{2 \sqrt{t}} .
\end{aligned}
$$

\section{REFERENCES}

[1] Stewartson, K. (1951). On the Impulsive Motion of a Flat Plate in a Viscous Fluid. Part 1, Quart. Journal. Mech. Appl. Math., 4, 2, pp. 182-198.

[2] Stewartson, K. (1973). On the Impulsive Motion of a Flat Plate in a Viscous Fluid. Part 2, Quart. Journal. Mech. Appl.Math., 22, 2, pp. 143-152. 
[3] Prasad, V.R., Bhaskar Reddy N. and Muthucumaraswamy R. (2007). Mass transfer and radiation effects on 2-D flow past an impulsively started infinite vertical plate. International. J.of Thermal Sciences 46, 1251-1258.

[4] Ismail, Z., Khan, I., Imran, A., Hussanan,A., Sharidan, S.(2014). MHD double diffusion flow by free convection past an infinite inclined plate with ramped wall temperature in a porous medium Malaysian J. of Fundamental and App. Sci. Vol.10, No.1.

[5] Chamka, A. J. (2003). MHD flow of a uniformly stretched vertical permeable surface in the presence of heat absorption /generation and chemical reaction. Int.Communication Heat Mass Transfer 3, $413-422$.

[6] Makinde, O.D. and Mhone, P.Y. (2005). Heat transfer to MHD oscillatory flow in a channel filled with porous medium. Romania Journal of Physics50(9-10), 931-938.

[7] Agarwal, H.L., Ram, P.C. and Singh, V.( 1983). Combined effect of dissipation and Hall effect on free convective in a rotating fluid. Indian J. Pure ppl. Math. 14(3):314-32 .

[8] Mazumdar B.S., Gupta A.S. and dattaa N. (1976). Flow and heat transfer inhydrodynamic ekman layer on a porous plate with Hall effects, Int.J.heat mass Transfer,19,523.

[9] Jaimala, Vikrant and Kumar Vivek. (2013). Thermal Convection in a C-S Fluid in the Presence of Horizontal Magnetic Field with Hall currents. Application and applied Mathematics, Vol.8, Issue, pp. 161-117.

[10]Reddy, M.G. (2014). Heat \& mass transfer effects on unsteady MHD radiative flow of a chemically reacting fluid past an impulsively started vertical plate, MATEMATIKA, Volume 30, Number 1, 1-15, UTM Centre for Industrial and Applied Mathematics.

[11]Mahmoudpour , M.E. and Rouzbahani, F. (2016). MHD free convection flow of a non-newtonian power-law fluid over a vertical plate with suction effects. J. Fundam Appl.Sci.,8(2S), 487-500.

[12]Brewster, M.Q.(1992). Thermal Radiative Transfer and Properties. John Wiley \& Sons, New York. 


\section{How to cite this article:}

Rajput US and Shareef M. Unsteady MHD flow along exponentially accelerated vertical flat surface through porous medium with variable temperature and hall current in a roatating system. J. Fundam. Appl. Sci., 2017, 9(2), 1050-1062. 